\title{
Generation of an Annual Typical Daily Wind Speed for Heights Equal and Less than 10 meters for Urban Armidale NSW, Australia
}

\author{
Yasser Maklad ${ }^{1}$ \\ ${ }^{\text {I}}$ (University of New England, Armidale NSW 2351 NSW Australia-School of Environmental \& Rural Science - \\ email: ymaklad@myune.edu.au)
}

\begin{abstract}
Wind has a very intermittent nature, its behaviour and speed are directly affected by several factors, specifically the nature of the environment and the height that wind blows at. Wind behaviour at urban large city areas and urban suburban areas is completely different than such behaviour at open terrain and flat unobstructed areas, wind turns to be more disturbed and turbulent and wind speed is reduced due to the existence of several obstructions, mostly buildings either single storey or medium rise or high rise buildings in addition to trees. As well, the height at which wind is blowing, the lower height the more disturbed wind the less wind speed, such effect is maximised in urban areas and near to the ground level. This paper aims to estimate and generate, in urban Armidale NSW, Australia, an annual typical daily wind speed for heights equal and less than $10 \mathrm{~m}$. A numerical method is applied to calculate such wind speed at urban Armidale at various heights $(10,9,8,7,6,5 \& 4) \mathrm{m}$ utilising an already previously generated typical wind speed reference year (TRY) of Armidale based on historical meteorological wind speed records measured at $10 \mathrm{~m}$ height at Armidale's Airport Weather Station (AWS) over a period of 20 years (1994 to 2013). Achieving such a database of typical reference year of daily wind speed at heights equal and less than 10 meters in $(\mathrm{m} / \mathrm{s})$ is an added value to the micro-scale wind energy generation systems, ranges from $0.5 \mathrm{~kW}$ to $10 \mathrm{~kW}$, as such systems involves micro-scale wind turbines would be operating at heights considered at this study.
\end{abstract}

Keywords: - Armidale NSW, test meteorological year, test reference year, wind speed, micro-scale energy generation, micro-wind turbines

\section{INTRODUCTION}

When wind flows over an open area approaches the boundaries of the built-up area, it encounters a high roughness of the surface, created by the buildings. The increased resistance resulting from this roughness reduces the wind flow at the level of the urban canopy. In this way a transitional zone is created between the ground and the undisturbed wind flow above the urban air dome, which is called "urban boundary layer". The "undisturbed flow" is called the "gradient wind" and its velocity is called the gradient velocity. Near the ground, wind experiences friction. Its speed is reduced more steeply and its turbulence increases. [1, 2 \&3].

The main urban design elements which can modify the wind conditions are: the overall density of the urban area, size and height of the individual buildings; existence of high-rise buildings, orientation of the streets, availability, size distribution, and design details of open spaces and green shelter belts. [4, 5 \& 6].

The wind field is characterized by two parameters: the vertical profile of the mean wind speed and the turbulence spectrum. Both are affected and modified by the profile of the terrain and, in an urban setup, by the urban structure $[4,5,6 \& 7]$.

Micro-scale energy generation ranges from [0.5 to 10$] \mathrm{kW}$ which suits domestic/residential, educational and small commercial applications., wind is one of the renewable inexhaustible energy sources for this scale of energy generation. Thus it most likely that micro scale wind turbines would operate at level higher than a house or a school or shop building.

This study focuses on the generation of wind speed at urban Armidale NSW, Australia at heights $(10,9,8,7,6$, $5 \& 4)]$ meters. This scope is selected mainly to provide micro-scale wind energy generation systems' designers an annual realistic mean daily speed such heights of micro-wind turbines' possible installations.

\section{DATA AND LOCATION}

A previously meteorological wind speed TRY at 10m height was developed [8]. That TRY was generated based on the daily mean wind speed recorded during the period 1994-2010 recorded at Armidale's Airport Weather Station (AWS). That TYR was generated utilising Finkelstein-Schafer (FS) statistics [9].

In Australia, meteorological observations are recorded by the Australian Bureau of Meteorology (BOM) weather stations are widely spreader in lots of cities and towns around Australia. In this study, the global wind 
speed data recorded by Armidale Airport Weather Automatic Station and published on the BOM's website where it was collected. The missing and invalid measurements account for $0.001 \%$ of the whole database of mean wind speed; those were replaced with the values of preceding or subsequent days by interpolation. During the calculations process, any year found with more than ten days in any month observations not available was excluded. "Table 1" provides geographical information for Armidale town and the periods of the relevant mean wind speed data and "figure 1" shows Armidale's location in NSW, Australia.

Table 1 Geographical and mean wind speed database information of Armidale NSW, Australia Longitude ( †E) Latitude $(\dagger \mathrm{S})$ Elevation $(\mathrm{m}) \quad$ Mean Daily Wind Speed

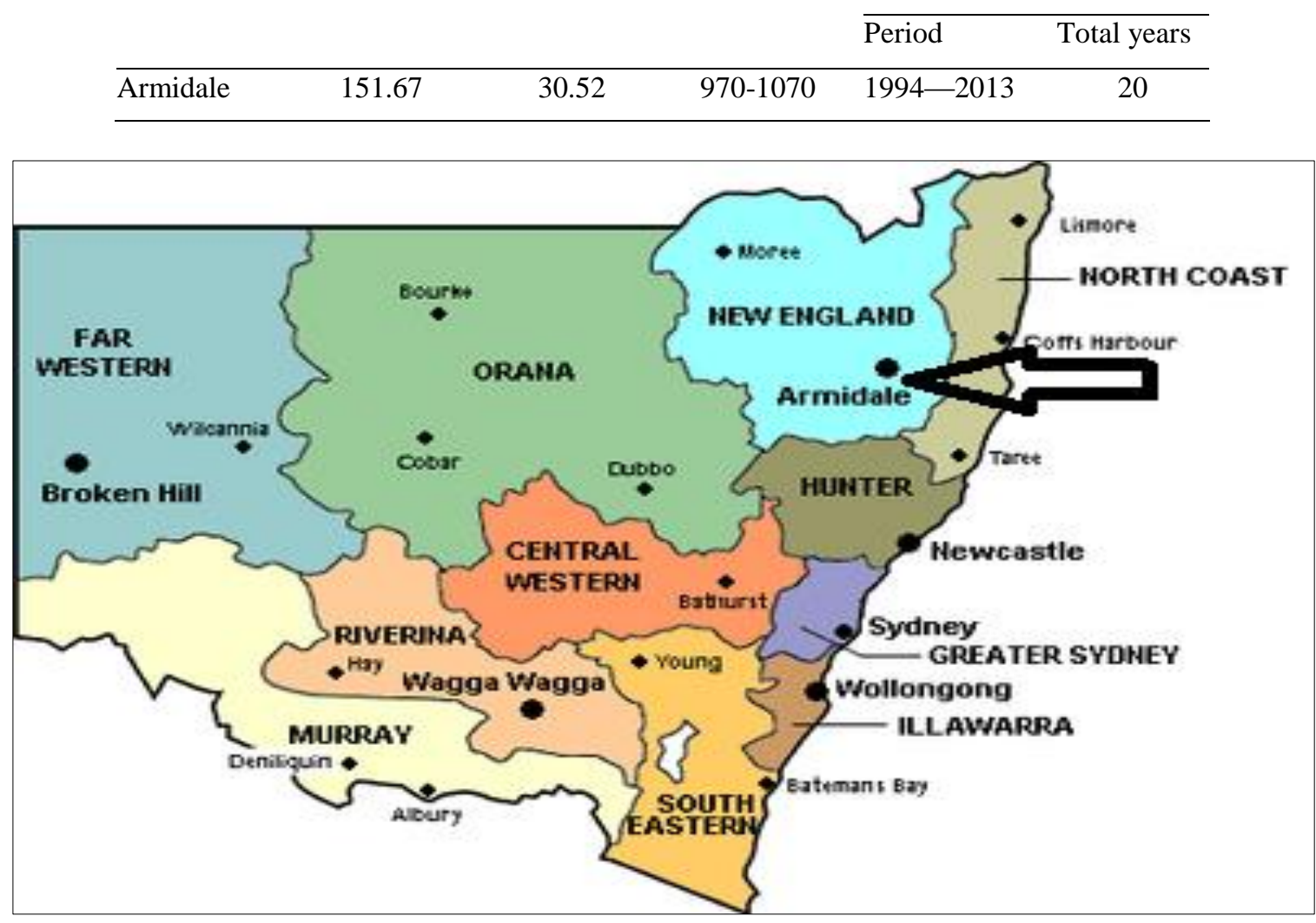

Figure1 Armidale NSW, Australia location

\section{METHODOLOGY}

To estimate the wind speed in urban areas, a reference measured wind speed is required which is usually the wind speeds measured and recorded by meteorological stations at a specified height. Such height is usually ten meters from ground level. Although, meteorological stations give all needed data but the problem is that the terrain of the station area and the height of the wind anemometer, are different from the design site [10]

The information of wind data in meteorology stations is gotten in the height of 10 meters above the ground and in a flat and completely open area. In lower levels the wind speed will be reduced because of the friction of the earth surfaces. The amount of the wind speed reduction depends on the terrain conditions. Reduction of the wind speed on the large area of water surface is the least and in the compressed urban area with low and high rise buildings is the most (Table 2). So the data of the wind speed reported by the meteorology station is not enough to estimate the wind speed in urban areas [10].

A numerical method is presented, in modelling the urban effect on the wind speed, the vertical profile of the wind, from the gradient wind level down to the ground is used. The simple equation developed by Davenport in 1960 [5] - shows the profile of the wind speed in different height of an area. It shows that the wind speed vary with height according to a power low [11].

$$
\frac{\overline{V_{Z}}}{\overline{V_{G}}}=\left[\frac{Z}{Z_{G}}\right]^{-\alpha}
$$

Where: 
$\overline{V_{Z}}=$ mean wind speed at height $\mathrm{Z}$ in the study site $(\mathrm{m} / \mathrm{s})$

$\overline{V_{G}}=$ mean wind speed at height $Z_{G}$ ("gradient" height) at top of the boundary layer of the study site, above which the speed is assumed to be constant, $(\mathrm{m} / \mathrm{s})$

$\mathrm{Z}=$ the height for which the wind speed $z V$ is computed $(\mathrm{m})$

$Z_{G}=$ the height at which "gradient velocity" $\overline{V_{G}}$ is first observed (layer thickness) in the same terrain (m)

$\alpha=$ an empirical exponent which depends on the surface roughness, stability and temperature gradient

"Table 2" shows the typical values of $Z_{G}$ and $\alpha$ for mean wind speeds over four types of terrain.

Table 2 Suggested Values of $Z_{G}$ and $\alpha$ for Various Terrain Conditions

\begin{tabular}{|c|l|c|c|}
\hline $\begin{array}{c}\text { Terrain } \\
\text { Category }\end{array}$ & \multicolumn{1}{|c|}{ Description } & Exponent $\alpha$ & $\begin{array}{c}\text { Layer Thickness } \\
Z_{G} \mathrm{~m}\end{array}$ \\
\hline 1 & $\begin{array}{l}\text { Large city centers, in which at least 50\% } \\
\text { of buildings are higher than 21 m, over a } \\
\text { distance of at least 2000 meters upwind. }\end{array}$ & 0.33 & 460 \\
\hline 2 & $\begin{array}{l}\text { Urban, suburban, wooded areas, and } \\
\text { other areas with closely spaced } \\
\text { obstructions compared to or larger than } \\
\text { single-family dwellings (over a distance } \\
\text { of at least 2000meters upwind). }\end{array}$ & 0.22 & 370 \\
\hline 3 & $\begin{array}{l}\text { Open terrain with scattered obstacles } \\
\text { generally less than 10 meters height. }\end{array}$ & 0.14 & 270 \\
\hline 4 & $\begin{array}{l}\text { Flat, unobstructed areas exposed to wind } \\
\text { flowing over a large water body (no more } \\
\text { than 500 meters inland). }\end{array}$ & 0.1 & 210 \\
\hline
\end{tabular}

Equation (1) will be developed to equation (2) which helps to calculate wind speed in the same terrain for every study height.

Where:

$$
\frac{\overline{V_{Z}}}{\overline{V_{Z 10}}}=\left[\frac{Z}{Z_{10}}\right]^{\alpha}
$$

$\overline{V_{Z 10}}=$ mean wind speed at the height of 10 meters in the study terrain $(\mathrm{m} / \mathrm{s})$

$\mathrm{Z}=$ the study height above the ground in the same terrain $(\mathrm{m})$

$Z_{10}=$ the height of 10 meters in the same terrain $(\mathrm{m})$

To estimate the wind speed in different urban areas with different density and terrain roughness, equation (3) is introduced:

where:

$$
\frac{\overline{V_{m e t 10}}}{\overline{V_{Z 10}}}=\frac{\left[\frac{m e t_{10}}{m e t_{G}}\right]^{\alpha_{m e t}}}{\left[\frac{Z_{10}}{Z_{G}}\right]^{\alpha}}
$$

$\overline{V_{\text {met }_{10}}}=$ mean wind speed at height of 10 meters in the meteorology station $(\mathrm{m} / \mathrm{s})$

met $_{101}=$ the standard observation height of 10 meters in the meteorology station $(\mathrm{m})$

$Z_{G}=$ gradient height at the top of the boundary layer of the study terrain

\section{GENERATION OF TYPICAL WIND SPEED YEAR}

"Table 3" shows the meteorological TRY for wind speed measured at 10 meter height in $(\mathrm{m} / \mathrm{s})$

Applying the above methodology for all the months in the database, the meteorological Test Reference Year for daily wind speed data was formed for Armidale. " Tables 4, 5, 6, 7, 8, 9 \& 10 " shows the calculated urban daily wind speed values obtained from Test Reference Year data for Armidale NSW, Australia at heights $(10,9,8,7,6$, $5 \& 4)$ respectively in $(\mathrm{m} / \mathrm{s})$. 
Generation of an Annual Typical Daily Wind Speed for Heights Equal and Less than 10 meters for Urban Armidale NSW, Australia

Table 3 Meteorological daily wind speed values obtained from Test Reference Year data for Armidale NSW, Australia at $10 \mathrm{~m}$ height in $(\mathrm{m} / \mathrm{s})$

\begin{tabular}{|c|c|c|c|c|c|c|c|c|c|c|c|c|}
\hline Day & Jan & Feb & Mar & Apr & May & Jun & Jul & Aug & Sep & Oct & Nov & Dec \\
\hline 1 & 5.59 & 6.29 & 5.94 & 5 & 5.14 & 4.59 & 6.04 & 5.63 & 5.31 & 6.04 & 5.63 & 5.31 \\
\hline 2 & 6.49 & 6.74 & 5.86 & 5.21 & 5.31 & 5.24 & 5.31 & 5.86 & 5.08 & 5.7 & 5.86 & 5.73 \\
\hline 3 & 5.59 & 5.94 & 5.86 & 4.73 & 4.86 & 4.51 & 5.21 & 6.84 & 6.35 & 5.39 & 6.04 & 5.7 \\
\hline 4 & 6.53 & 5.45 & 6.11 & 4.76 & 5.39 & 4.1 & 5.7 & 6.11 & 5.8 & 4.96 & 5.31 & 6.04 \\
\hline 5 & 5.86 & 5.04 & 6.33 & 5.63 & 4.51 & 5.08 & 5.7 & 6.53 & 6.21 & 4.83 & 5.04 & 6.49 \\
\hline 6 & 5.9 & 5.41 & 6.35 & 5.73 & 4.96 & 5.04 & 5.49 & 6.25 & 6.25 & 6.21 & 5.49 & 6.25 \\
\hline 7 & 6.49 & 6.49 & 5.7 & 6.04 & 5.14 & 4.86 & 4.73 & 6.53 & 5.7 & 5.86 & 5.49 & 5.94 \\
\hline 8 & 6.56 & 5.63 & 5.14 & 5.7 & 4.41 & 5.39 & 5.21 & 6.84 & 6.11 & 6.35 & 5.86 & 5.41 \\
\hline 9 & 6.84 & 6.11 & 5.73 & 4.83 & 4.41 & 5.8 & 6.18 & 6.11 & 6.18 & 6.18 & 5.59 & 5.24 \\
\hline 10 & 6.11 & 5.49 & 5.63 & 5.18 & 4.73 & 5.49 & 5.94 & 6.18 & 5.49 & 5.55 & 5.63 & 5.94 \\
\hline 11 & 6.33 & 5.21 & 5.7 & 4.76 & 4.83 & 5.31 & 5.94 & 6.11 & 6.01 & 5.31 & 5.49 & 5.55 \\
\hline 12 & 5.94 & 5.7 & 5.55 & 5.49 & 4.86 & 4.83 & 4.51 & 6.6 & 5.55 & 5.55 & 5.63 & 5.18 \\
\hline 13 & 5.63 & 6.01 & 5.39 & 4.93 & 5.08 & 5.35 & 4.65 & 5.8 & 5.73 & 5.8 & 6.11 & 5.55 \\
\hline 14 & 5.63 & 6.35 & 5.08 & 4.28 & 5.14 & 6.21 & 6.04 & 6.49 & 5.21 & 6.25 & 5.21 & 5.86 \\
\hline 15 & 5.94 & 6.04 & 4.41 & 4.34 & 4.2 & 5.66 & 5.7 & 6.04 & 5.21 & 5.39 & 5.8 & 5.49 \\
\hline 16 & 5.94 & 5.39 & 4.9 & 4.65 & 5.08 & 5.21 & 4.51 & 6.53 & 8.85 & 6.56 & 6.11 & 5.08 \\
\hline 17 & 5.94 & 5.49 & 5.14 & 4.96 & 5.18 & 5.24 & 4.65 & 6.18 & 6.35 & 6.29 & 5.94 & 5.18 \\
\hline 18 & 5.94 & 6.11 & 5.84 & 4.65 & 4.86 & 4.73 & 5.31 & 6.66 & 5.31 & 5.63 & 5.49 & 5.8 \\
\hline 19 & 6.43 & 5.53 & 5.35 & 4.2 & 5.8 & 5.55 & 5.7 & 5.63 & 5.28 & 5.7 & 4.96 & 5.63 \\
\hline 20 & 6.11 & 5.7 & 5.21 & 4.34 & 5.14 & 5.18 & 5.39 & 6.11 & 5.39 & 5.39 & 5.31 & 5.94 \\
\hline 21 & 5.94 & 5.55 & 4.9 & 4.2 & 5.53 & 6.21 & 6.6 & 6.35 & 5.08 & 4.51 & 5.55 & 5.39 \\
\hline 22 & 5.45 & 5.8 & 5.39 & 4.41 & 4.93 & 6.11 & 5.14 & 6.84 & 4.34 & 5.63 & 6.11 & 5.45 \\
\hline 23 & 5.24 & 6.04 & 5.35 & 4.41 & 4.51 & 5.9 & 5.39 & 5.86 & 5.8 & 6.18 & 5.94 & 5.55 \\
\hline 24 & 6.25 & 5.94 & 5.21 & 4.83 & 4.65 & 5.14 & 5.08 & 6.6 & 5.7 & 5.39 & 5.31 & 5.86 \\
\hline 25 & 6.43 & 5.8 & 5.24 & 5.31 & 4.73 & 4.59 & 5.31 & 6.25 & 6.11 & 5.39 & 5.1 & 5.39 \\
\hline 26 & 6.01 & 5.14 & 5.04 & 5.45 & 5.21 & 5.24 & 6.49 & 6.18 & 6.04 & 6.15 & 5.08 & 5.41 \\
\hline 27 & 6.11 & 4.76 & 4.51 & 5.31 & 4.83 & 5.31 & 5.86 & 6.11 & 6.25 & 6.25 & 6.35 & 5.45 \\
\hline 28 & 5.94 & 5 & 4.83 & 5.53 & 4.79 & 6.6 & 6.11 & 6.21 & 6.11 & 5.14 & 5.94 & 5.21 \\
\hline 29 & 5.73 & 5.55 & 4.59 & 5.24 & 5.49 & 6.84 & 5.63 & 6.01 & 6.88 & 5.98 & 5.45 & 5.49 \\
\hline 30 & 5.49 & & 4.59 & 5.04 & 4.69 & 6.04 & 5.49 & 7.4 & 5.39 & 5.49 & 5.55 & 5.84 \\
\hline 31 & 6.25 & & 5 & & 4.9 & & 5.49 & 6.64 & & 6.11 & & 5.7 \\
\hline
\end{tabular}


Generation of an Annual Typical Daily Wind Speed for Heights Equal and Less than 10 meters for Urban Armidale NSW, Australia

Table 4 Urban daily wind speed values obtained from Test Reference Year data for Armidale NSW, Australia at $10 \mathrm{~m}$ height in $(\mathrm{m} / \mathrm{s})$

\begin{tabular}{|c|c|c|c|c|c|c|c|c|c|c|c|c|}
\hline Day & Jan & Feb & Mar & Apr & May & Jun & Jul & Aug & Sep & Oct & Nov & Dec \\
\hline 1 & 4.01 & 4.51 & 4.26 & 3.58 & 3.68 & 3.29 & 4.33 & 4.04 & 3.81 & 4.33 & 4.04 & 3.81 \\
\hline 2 & 4.65 & 4.83 & 4.2 & 3.73 & 3.81 & 3.76 & 3.81 & 4.2 & 3.64 & 4.09 & 4.2 & 4.11 \\
\hline 3 & 4.01 & 4.26 & 4.2 & 3.39 & 3.48 & 3.23 & 3.73 & 4.9 & 4.55 & 3.86 & 4.33 & 4.09 \\
\hline 4 & 4.68 & 3.91 & 4.38 & 3.41 & 3.86 & 2.94 & 4.09 & 4.38 & 4.16 & 3.56 & 3.81 & 4.33 \\
\hline 5 & 4.2 & 3.61 & 4.54 & 4.04 & 3.23 & 3.64 & 4.09 & 4.68 & 4.45 & 3.46 & 3.61 & 4.65 \\
\hline 6 & 4.23 & 3.88 & 4.55 & 4.11 & 3.56 & 3.61 & 3.94 & 4.48 & 4.48 & 4.45 & 3.94 & 4.48 \\
\hline 7 & 4.65 & 4.65 & 4.09 & 4.33 & 3.68 & 3.48 & 3.39 & 4.68 & 4.09 & 4.2 & 3.94 & 4.26 \\
\hline 8 & 4.7 & 4.04 & 3.68 & 4.09 & 3.16 & 3.86 & 3.73 & 4.9 & 4.38 & 4.55 & 4.2 & 3.88 \\
\hline 9 & 4.9 & 4.38 & 4.11 & 3.46 & 3.16 & 4.16 & 4.43 & 4.38 & 4.43 & 4.43 & 4.01 & 3.76 \\
\hline 10 & 4.38 & 3.94 & 4.04 & 3.71 & 3.39 & 3.94 & 4.26 & 4.43 & 3.94 & 3.98 & 4.04 & 4.26 \\
\hline 11 & 4.54 & 3.73 & 4.09 & 3.41 & 3.46 & 3.81 & 4.26 & 4.38 & 4.31 & 3.81 & 3.94 & 3.98 \\
\hline 12 & 4.26 & 4.09 & 3.98 & 3.94 & 3.48 & 3.46 & 3.23 & 4.73 & 3.98 & 3.98 & 4.04 & 3.71 \\
\hline 13 & 4.04 & 4.31 & 3.86 & 3.53 & 3.64 & 3.83 & 3.33 & 4.16 & 4.11 & 4.16 & 4.38 & 3.98 \\
\hline 14 & 4.04 & 4.55 & 3.64 & 3.07 & 3.68 & 4.45 & 4.33 & 4.65 & 3.73 & 4.48 & 3.73 & 4.2 \\
\hline 15 & 4.26 & 4.33 & 3.16 & 3.11 & 3.01 & 4.06 & 4.09 & 4.33 & 3.73 & 3.86 & 4.16 & 3.94 \\
\hline 16 & 4.26 & 3.86 & 3.51 & 3.33 & 3.64 & 3.73 & 3.23 & 4.68 & 6.34 & 4.7 & 4.38 & 3.64 \\
\hline 17 & 4.26 & 3.94 & 3.68 & 3.56 & 3.71 & 3.76 & 3.33 & 4.43 & 4.55 & 4.51 & 4.26 & 3.71 \\
\hline 18 & 4.26 & 4.38 & 4.19 & 3.33 & 3.48 & 3.39 & 3.81 & 4.77 & 3.81 & 4.04 & 3.94 & 4.16 \\
\hline 19 & 4.61 & 3.96 & 3.83 & 3.01 & 4.1 & 3.98 & 4.09 & 4.04 & 3.78 & 4.09 & 3.56 & 4.04 \\
\hline 20 & 4.38 & 4.09 & 3.73 & 3.11 & 3.68 & 3.71 & 3.86 & 4.38 & 3.86 & 3.86 & 3.81 & 4.26 \\
\hline 21 & 4.26 & 3.98 & 3.51 & 3.01 & 3.96 & 4.45 & 4.73 & 4.55 & 3.64 & 3.23 & 3.98 & 3.86 \\
\hline 22 & 3.91 & 4.16 & 3.86 & 3.16 & 3.53 & 4.38 & 3.68 & 4.9 & 3.11 & 4.04 & 4.38 & 3.91 \\
\hline 23 & 3.76 & 4.33 & 3.83 & 3.16 & 3.23 & 4.23 & 3.86 & 4.2 & 4.16 & 4.43 & 4. & 3.98 \\
\hline 24 & 4.48 & 4.26 & 3.73 & 3.46 & 3.33 & 3.68 & 3.64 & 4.73 & 4.09 & 3.86 & 3.81 & 4.2 \\
\hline 25 & 4.61 & 4.16 & 3.76 & 3.81 & 3.39 & 3.29 & 3.81 & 4.48 & 4.38 & 3.86 & 3.66 & 3.86 \\
\hline 26 & 4.31 & 3.68 & 3.61 & 3.91 & 3.73 & 3.76 & 4.65 & 4.43 & 4.33 & 4.41 & 3.64 & 3.88 \\
\hline 27 & 4.38 & 3.41 & 3.23 & 3.81 & 3.46 & 3.81 & 4.2 & 4.38 & 4.48 & 4.48 & 4.55 & 3.91 \\
\hline 28 & 4.26 & 3.58 & 3.46 & 3.96 & 3.4 & 4.73 & 4.38 & 4.45 & 4.38 & 3.68 & 4.26 & 3.73 \\
\hline 29 & 4.11 & 3.98 & 3.29 & 3.76 & 3.94 & 4.9 & 4.04 & 4.31 & 4.93 & 4.29 & 3.91 & 3.94 \\
\hline 30 & 3.94 & & 3.29 & 3.61 & 3.36 & 4.33 & 3.94 & 5.3 & 3.86 & 3.94 & 3.98 & 4.19 \\
\hline 31 & 4.48 & & 3.58 & & 3.51 & & 3.94 & 4.76 & & 4.38 & & 4.09 \\
\hline
\end{tabular}


Generation of an Annual Typical Daily Wind Speed for Heights Equal and Less than 10 meters for Urban Armidale NSW, Australia

Table 5 Urban daily wind speed values obtained from Test Reference Year data for Armidale NSW, Australia at $9 \mathrm{~m}$ height in $(\mathrm{m} / \mathrm{s})$

\begin{tabular}{|c|c|c|c|c|c|c|c|c|c|c|c|c|}
\hline Day & Jan & Feb & Mar & Apr & May & Jun & Jul & Aug & Sep & Oct & Nov & Dec \\
\hline 1 & 3.92 & 4.41 & 4.16 & 3.5 & 3.6 & 3.21 & 4.23 & 3.95 & 3.72 & 4.23 & 3.95 & 3.72 \\
\hline 2 & 4.54 & 4.72 & 4.1 & 3.64 & 3.72 & 3.67 & 3.72 & 4.1 & 3.56 & 4 & 4.1 & 4.02 \\
\hline 3 & 3.92 & 4.16 & 4.1 & 3.31 & 3.4 & 3.16 & 3.64 & 4.79 & 4.45 & 3.77 & 4.23 & 4 \\
\hline 4 & 4.57 & 3.82 & 4.28 & 3.33 & 3.77 & 2.87 & 4 & 4.28 & 4.06 & 3.48 & 3.72 & 4.23 \\
\hline 5 & 4.1 & 3.53 & 4.44 & 3.95 & 3.16 & 3.56 & 4 & 4.57 & 4.35 & 3.38 & 3.53 & 4.54 \\
\hline 6 & 4.13 & 3.79 & 4.45 & 4.02 & 3.48 & 3.53 & 3.85 & 4.38 & 4.38 & 4.35 & 3.85 & 4.38 \\
\hline 7 & 4.54 & 4.54 & 4 & 4.23 & 3.6 & 3.4 & 3.31 & 4.57 & 4 & 4.1 & 3.85 & 4.16 \\
\hline 8 & 4.59 & 3.95 & 3.6 & 4 & 3.09 & 3.77 & 3.64 & 4.79 & 4.28 & 4.45 & 4.1 & 3.79 \\
\hline 9 & 4.79 & 4.28 & 4.02 & 3.38 & 3.09 & 4.06 & 4.33 & 4.28 & 4.33 & 4.33 & 3.92 & 3.67 \\
\hline 10 & 4.28 & 3.85 & 3.95 & 3.62 & 3.31 & 3.85 & 4.16 & 4.33 & 3.85 & 3.89 & 3.95 & 4.16 \\
\hline 11 & 4.44 & 3.64 & 4 & 3.33 & 3.38 & 3.72 & 4.16 & 4.28 & 4.21 & 3.72 & 3.85 & 3.89 \\
\hline 12 & 4.16 & 4 & 3.89 & 3.85 & 3.4 & 3.38 & 3.16 & 4.62 & 3.89 & 3.89 & 3.95 & 3.62 \\
\hline 13 & 3.95 & 4.21 & 3.77 & 3.45 & 3.56 & 3.74 & 3.25 & 4.06 & 4.02 & 4.06 & 4.28 & 3.89 \\
\hline 14 & 3.95 & 4.45 & 3.56 & 3 & 3.6 & 4.35 & 4.23 & 4.54 & 3.64 & 4.38 & 3.64 & 4.1 \\
\hline 15 & 4.16 & 4.23 & 3.09 & 3.04 & 2.94 & 3.97 & 4 & 4.23 & 3.64 & 3.77 & 4.06 & 3.85 \\
\hline 16 & 4.16 & 3.77 & 3.43 & 3.25 & 3.56 & 3.64 & 3.16 & 4.57 & 6.19 & 4.59 & 4.28 & 3.56 \\
\hline 17 & 4.16 & 3.85 & 3.6 & 3.48 & 3.62 & 3.67 & 3.25 & 4.33 & 4.45 & 4.41 & 4.16 & 3.62 \\
\hline 18 & 4.16 & 4.28 & 4.09 & 3.25 & 3.4 & 3.31 & 3.72 & 4.66 & 3.72 & 3.95 & 3.85 & 4.06 \\
\hline 19 & 4.5 & 3.87 & 3.74 & 2.94 & 4.06 & 3.89 & 4 & 3.95 & 3.69 & 4 & 3.48 & 3.95 \\
\hline 20 & 4.28 & 4 & 3.64 & 3.04 & 3.6 & 3.62 & 3.77 & 4.28 & 3.77 & 3.77 & 3.72 & 4.16 \\
\hline 21 & 4.16 & 3.89 & 3.43 & 2.94 & 3.87 & 4.35 & 4.62 & 4.45 & 3.56 & 3.16 & 3.89 & 3.77 \\
\hline 22 & 3.82 & 4.06 & 3.77 & 3.09 & 3.45 & 4.28 & 3.6 & 4.79 & 3.04 & 3.95 & 4.28 & 3.82 \\
\hline 23 & 3.67 & 4.23 & 3.74 & 3.09 & 3.16 & 4.13 & 3.77 & 4.1 & 4.06 & 4.33 & 4.16 & 3.89 \\
\hline 24 & 4.38 & 4.16 & 3.64 & 3.38 & 3.25 & 3.6 & 3.56 & 4.62 & 4 & 3.77 & 3.72 & 4.1 \\
\hline 25 & 4.5 & 4.06 & 3.67 & 3.72 & 3.31 & 3.21 & 3.72 & 4.38 & 4.28 & 3.77 & 3.58 & 3.77 \\
\hline 26 & 4.21 & 3.6 & 3.53 & 3.82 & 3.64 & 3.67 & 4.54 & 4.33 & 4.23 & 4.31 & 3.56 & 3.79 \\
\hline 27 & 4.28 & 3.33 & 3.16 & 3.72 & 3.38 & 3.72 & 4.1 & 4.28 & 4.38 & 4.38 & 4.45 & 3.82 \\
\hline 28 & 4.16 & 3.5 & 3.38 & 3.87 & 3.35 & 4.62 & 4.28 & 4.35 & 4.28 & 3.6 & 4.16 & 3.64 \\
\hline 29 & 4.02 & 3.89 & 3.21 & 3.67 & 3.85 & 4.79 & 3.95 & 4.21 & 4.82 & 4.19 & 3.82 & 3.85 \\
\hline 30 & 3.85 & & 3.21 & 3.53 & 3.28 & 4.23 & 3.85 & 5.18 & 3.77 & 3.85 & 3.89 & 4.09 \\
\hline 31 & 4.38 & & 3.5 & & 3.43 & & 3.85 & 4.65 & & 4.28 & & 4 \\
\hline
\end{tabular}


Generation of an Annual Typical Daily Wind Speed for Heights Equal and Less than 10 meters for Urban Armidale NSW, Australia

Table 6 Urban daily wind speed values obtained from Test Reference Year data for Armidale NSW, Australia at $8 \mathrm{~m}$ height in $(\mathrm{m} / \mathrm{s})$

\begin{tabular}{|c|c|c|c|c|c|c|c|c|c|c|c|c|}
\hline Day & Jan & Feb & Mar & Apr & May & Jun & Jul & Aug & Sep & Oct & Nov & Dec \\
\hline 1 & 3.82 & 4.29 & 4.06 & 3.41 & 3.5 & 3.13 & 4.12 & 3.85 & 3.63 & 4.12 & 3.85 & 3.63 \\
\hline 2 & 4.43 & 4.6 & 4 & 3.55 & 3.63 & 3.58 & 3.63 & 4 & 3.47 & 3.89 & 4 & 3.91 \\
\hline 3 & 3.82 & 4.06 & 4 & 3.23 & 3.31 & 3.08 & 3.55 & 4.67 & 4.33 & 3.68 & 4.12 & 3.89 \\
\hline 4 & 4.46 & 3.72 & 4.17 & 3.25 & 3.68 & 2.8 & 3.89 & 4.17 & 3.96 & 3.39 & 3.63 & 4.12 \\
\hline 5 & 4 & 3.44 & 4.32 & 3.85 & 3.08 & 3.47 & 3.89 & 4.46 & 4.24 & 3.29 & 3.44 & 4.43 \\
\hline 6 & 4.03 & 3.69 & 4.33 & 3.91 & 3.39 & 3.44 & 3.75 & 4.27 & 4.27 & 4.24 & 3.75 & 4.27 \\
\hline 7 & 4.43 & 4.43 & 3.89 & 4.12 & 3.5 & 3.31 & 3.23 & 4.46 & 3.89 & 4 & 3.75 & 4.06 \\
\hline 8 & 4.47 & 3.85 & 3.5 & 3.89 & 3.01 & 3.68 & 3.55 & 4.67 & 4.17 & 4.33 & 4 & 3.69 \\
\hline 9 & 4.67 & 4.17 & 3.91 & 3.29 & 3.01 & 3.96 & 4.22 & 4.17 & 4.22 & 4.22 & 3.82 & 3.58 \\
\hline 10 & 4.17 & 3.75 & 3.85 & 3.53 & 3.23 & 3.75 & 4.06 & 4.22 & 3.75 & 3.79 & 3.85 & 4.06 \\
\hline 11 & 4.32 & 3.55 & 3.89 & 3.25 & 3.29 & 3.63 & 4.06 & 4.17 & 4.1 & 3.63 & 3.75 & 3.79 \\
\hline 12 & 4.06 & 3.89 & 3.79 & 3.75 & 3.31 & 3.29 & 3.08 & 4.5 & 3.79 & 3.79 & 3.85 & 3.53 \\
\hline 13 & 3.85 & 4.1 & 3.68 & 3.36 & 3.47 & 3.65 & 3.17 & 3.96 & 3.91 & 3.96 & 4.17 & 3.79 \\
\hline 14 & 3.85 & 4.33 & 3.47 & 2.92 & 3.5 & 4.24 & 4.12 & 4.43 & 3.55 & 4.27 & 3.55 & 4 \\
\hline 15 & 4.06 & 4.12 & 3.01 & 2.96 & 2.87 & 3.87 & 3.89 & 4.12 & 3.55 & 3.68 & 3.96 & 3.75 \\
\hline 16 & 4.06 & 3.68 & 3.34 & 3.17 & 3.47 & 3.55 & 3.08 & 4.46 & 6.04 & 4.47 & 4.17 & 3.47 \\
\hline 17 & 4.06 & 3.75 & 3.5 & 3.39 & 3.5 & 3.58 & 3.17 & 4.22 & 4.33 & 4.2 & 4.06 & 3.53 \\
\hline 18 & 4.06 & 4.17 & 3.99 & 3.17 & 3.31 & 3.23 & 3.63 & 4.54 & 3.63 & 3.85 & 3.75 & 3.96 \\
\hline 19 & 4.39 & 3.77 & 3.65 & 2.87 & 3.96 & 3.79 & 3.89 & 3.85 & 3.6 & 3.89 & 3.39 & 3.85 \\
\hline 20 & 4.17 & 3.89 & 3.55 & 2.96 & 3.5 & 3.53 & 3.68 & 4.17 & 3. & 3. & 3. & 4.06 \\
\hline 21 & 4.06 & 3.79 & 3.34 & 2.87 & 3.7 & 4.24 & 4.5 & 4.33 & 3.47 & 3.08 & 3.79 & 3.68 \\
\hline 22 & 3.72 & 3.96 & 3.68 & 3.01 & 3.36 & 4.17 & 3.5 & 4.67 & 2.96 & 3.85 & 4.17 & 3.72 \\
\hline 23 & 3.58 & 4.12 & 3.65 & 3.01 & 3.08 & 4.03 & 3.68 & 4 & 3.96 & 4.22 & 4.06 & 3.79 \\
\hline 24 & 4.27 & 4.06 & 3.55 & 3.29 & 3.1 & 3.5 & 3.47 & 4.5 & 3.8 & 3.6 & 3.63 & 4 \\
\hline 25 & 4.39 & 3.96 & 3.58 & 3.63 & 3.23 & 3.13 & 3.63 & 4.27 & 4.17 & 3.68 & 3.48 & 3.68 \\
\hline 26 & 4.1 & 3.5 & 3.44 & 3.72 & 3.55 & 3.58 & 4.43 & 4.22 & 4.12 & 4.2 & 3.47 & 3.69 \\
\hline 27 & 4.17 & 3.25 & 3.08 & 3.63 & 3.29 & 3.63 & 4 & 4.17 & 4.27 & 4.27 & 4.33 & 3.72 \\
\hline 28 & 4.06 & 3.41 & 3.29 & 3.77 & 3.27 & 4.5 & 4.17 & 4.24 & 4.17 & 3.5 & 4.06 & 3.55 \\
\hline 29 & 3.91 & 3.79 & 3.13 & 3.58 & 3.75 & 4.67 & 3.85 & 4.1 & 4.69 & 4.08 & 3.72 & 3.75 \\
\hline 30 & 3.75 & & 3.13 & 3.44 & 3.2 & 4.12 & 3.75 & 5.05 & 3.68 & 3.75 & 3.79 & 3.99 \\
\hline 31 & 4.27 & & 3.41 & & 3.34 & & 3.75 & 4.53 & & 4.17 & & 3.89 \\
\hline
\end{tabular}


Generation of an Annual Typical Daily Wind Speed for Heights Equal and Less than 10 meters for Urban Armidale NSW, Australia

Table 7 Urban daily wind speed values obtained from Test Reference Year data for Armidale NSW, Australia at

\begin{tabular}{|c|c|c|c|c|c|c|c|c|c|c|c|c|}
\hline Day & Jan & Feb & Mar & $\mathrm{Apr}$ & May & Jun & Jul & Aug & Sep & Oct & Nov & $\mathrm{Dec}$ \\
\hline 1 & 3.71 & 4.17 & 3.94 & 3.31 & 3.4 & 3.04 & 4 & 3.74 & 3.52 & 4 & 3.74 & 3.52 \\
\hline 2 & 4.3 & 4.47 & 3.88 & 3.45 & 3.52 & 3.48 & 3.52 & 3.88 & 3.37 & 3.78 & 3.88 & 3.8 \\
\hline 3 & 3.71 & 3.94 & 3.88 & 3.13 & 3.22 & 2.99 & 3.45 & 4.53 & 4.21 & 3.57 & $\begin{array}{c}0.00 \\
4\end{array}$ & $\begin{array}{l}5.0 \\
3.78\end{array}$ \\
\hline 4 & 4.33 & 3.61 & 4.05 & 3.15 & 3.57 & 2.72 & 3.78 & 4.05 & 3.85 & 3.29 & 3.52 & 4 \\
\hline 5 & 3.88 & 3.34 & 4.2 & 3.74 & 2.99 & 3.37 & 3.78 & 4.33 & 4.11 & 3.2 & 3.34 & 4.3 \\
\hline 6 & 3.91 & 3.59 & 4.21 & 3.8 & 3.29 & 3.34 & 3.64 & 4.14 & 4.14 & 4.11 & 3.64 & 4.14 \\
\hline 7 & 4.3 & 4.3 & 3.78 & 4 & 3.4 & 3.22 & 3.13 & 4.33 & 3.78 & 3.88 & 3.64 & 3.94 \\
\hline 8 & 4.35 & 3.74 & 3.4 & 3.78 & 2.92 & 3.57 & 3.45 & 4.53 & 4.05 & 4.21 & 3.88 & 3.59 \\
\hline 9 & 4.53 & 4.05 & 3.8 & 3.2 & 2.92 & 3.85 & 4.1 & 4.05 & 4.1 & 4.1 & 3.71 & 3.48 \\
\hline 10 & 4.05 & 3.64 & 3.74 & 3.43 & 3.13 & 3.64 & 3.94 & 4.1 & 3.64 & 3.68 & 3.74 & 3.94 \\
\hline 11 & 4.2 & 3.45 & 3.78 & 3.15 & 3.2 & 3.52 & 3.94 & 4.05 & 3.98 & 3.52 & 3.64 & 3.68 \\
\hline 12 & 3.94 & 3.78 & 3.68 & 3.64 & 3.22 & 3.2 & 2.99 & 4.37 & 3.68 & 3.68 & 3.74 & 3.43 \\
\hline 13 & 3.74 & 3.98 & 3.57 & 3.26 & 3.37 & 3.54 & 3.08 & 3.85 & $\begin{array}{l}3.00 \\
3.8\end{array}$ & 3.85 & 4.05 & 3.68 \\
\hline 14 & 3.74 & 4.21 & 3.37 & 2.84 & 3.4 & 4.11 & 4 & 4.3 & 3.45 & 4.14 & 3.45 & 3.88 \\
\hline 15 & 3.94 & 4 & 2.92 & 2.88 & 2.78 & 3.75 & 3.78 & 4 & 3.45 & 3.57 & 3.85 & 3.64 \\
\hline 16 & 3.94 & 3.57 & 3.25 & 3.08 & 3.37 & 3.45 & 2.99 & 4.33 & 5.86 & 4.35 & 4.05 & 3.37 \\
\hline 17 & 3.94 & 3.64 & 3.4 & 3.29 & 3.43 & 3.48 & 3.08 & 4.1 & 4.21 & 4.17 & 3.94 & 3.43 \\
\hline 18 & 3.94 & 4.05 & 3.87 & 3.08 & 3.22 & 3.13 & 3.52 & 4.41 & 3.52 & 3.74 & 3.64 & 3.85 \\
\hline 19 & 4.26 & 3.66 & 3.54 & 2.78 & 3.85 & 3.68 & 3.78 & 3.74 & 3.49 & 3.78 & 3.29 & 3.74 \\
\hline 20 & 4.05 & 3.78 & 3.45 & 2.88 & 3.4 & 3.43 & 3.57 & 4.05 & 3.57 & 3.57 & 3.52 & 3.94 \\
\hline 21 & 3.94 & 3.68 & 3.25 & 2.78 & 3.66 & 4.11 & 4.37 & 4.21 & 3.37 & 2.99 & 3.68 & 3.57 \\
\hline 22 & 3.61 & 3.85 & 3.57 & 2.92 & 3.26 & 4.05 & 3.4 & 4.53 & 2.88 & 3.74 & 4.05 & 3.61 \\
\hline 23 & 3.48 & 4 & 3.54 & 2.92 & 2.99 & 3.91 & 3.57 & 3.88 & 3.85 & 4.1 & 3.94 & 3.68 \\
\hline 24 & 4.14 & 3.94 & 3.45 & 3.2 & 3.08 & 3.4 & 3.37 & 4.37 & 3.78 & 3.57 & 3.52 & 3.88 \\
\hline 25 & 4.26 & 3.85 & 3.48 & 3.52 & 3.13 & 3.04 & 3.52 & 4.14 & 4.05 & 3.57 & 3.38 & 3.57 \\
\hline 26 & 3.98 & 3.4 & 3.34 & 3.61 & 3.45 & 3.48 & 4.3 & 4.1 & 4 & 4.08 & 3.37 & 3.59 \\
\hline 27 & 4.05 & 3.15 & 2.99 & 3.52 & 3.2 & 3.52 & 3.88 & 4.05 & 4.14 & 4.14 & 4.21 & 3.61 \\
\hline 28 & 3.94 & 3.31 & 3.2 & 3.66 & 3.17 & 4.37 & 4.05 & 4.11 & 4.05 & 3.4 & 3.94 & 3.45 \\
\hline 29 & 3.8 & 3.68 & 3.04 & 3.48 & 3.64 & 4.53 & 3.74 & 3.98 & 4.56 & 3.97 & 3.61 & 3.64 \\
\hline 30 & 3.64 & & 3.04 & 3.34 & 3.11 & 4 & 3.64 & 4.9 & 3.57 & 3.64 & 3.68 & 3.87 \\
\hline 31 & 4.14 & & 3.31 & & 3.25 & & 3.64 & 4.4 & & 4.05 & & 3.78 \\
\hline
\end{tabular}


Generation of an Annual Typical Daily Wind Speed for Heights Equal and Less than 10 meters for Urban Armidale NSW, Australia

Table 8 Urban daily wind speed values obtained from Test Reference Year data for Armidale NSW, Australia at $6 \mathrm{~m}$ height in $(\mathrm{m} / \mathrm{s})$

\begin{tabular}{|c|c|c|c|c|c|c|c|c|c|c|c|c|}
\hline Day & Jan & Feb & Mar & Apr & May & Jun & Jul & Aug & Sep & Oct & Nov & Dec \\
\hline 1 & 3.58 & 4.03 & 3.81 & 3.2 & 3.29 & 2.94 & 3.87 & 3.61 & 3.41 & 3.87 & 3.61 & 3.41 \\
\hline 2 & 4.16 & 4.32 & 3.75 & 3.33 & 3.41 & 3.36 & 3.41 & 3.75 & 3.25 & 3.66 & 3.75 & 3.67 \\
\hline 3 & 3.58 & 3.81 & 3.75 & 3.03 & 3.11 & 2.89 & 3.33 & 4.38 & 4.07 & 3.45 & 3.87 & 3.66 \\
\hline 4 & 4.18 & 3.49 & 3.91 & 3.05 & 3.45 & 2.63 & 3.66 & 3.91 & 3.72 & 3.18 & 3.41 & 3.87 \\
\hline 5 & 3.75 & 3.23 & 4.06 & 3.61 & 2.89 & 3.25 & 3.66 & 4.18 & 3.98 & 3.09 & 3.23 & 4.16 \\
\hline 6 & 3.78 & 3.47 & 4.07 & 3.67 & 3.18 & 3.23 & 3.52 & 4 & 4 & 3.98 & 3.52 & 4 \\
\hline 7 & 4.16 & 4.16 & 3.66 & 3.87 & 3.29 & 3.11 & 3.03 & 4.18 & 3.66 & 3.75 & 3.52 & 3.81 \\
\hline 8 & 4.2 & 3.61 & 3.29 & 3.66 & 2.82 & 3.45 & 3.33 & 4.38 & 3.91 & 4.07 & 3.75 & 3.47 \\
\hline 9 & 4.38 & 3.91 & 3.67 & 3.09 & 2.82 & 3.72 & 3.96 & 3.91 & 3.96 & 3.96 & 3.58 & 3.36 \\
\hline 10 & 3.91 & 3.52 & 3.61 & 3.32 & 3.03 & 3.52 & 3.81 & 3.96 & 3.52 & 3.56 & 3.61 & 3.81 \\
\hline 11 & 4.06 & 3.33 & 3.66 & 3.05 & 3.09 & 3.41 & 3.81 & 3.91 & 3.85 & 3.41 & 3.52 & 3.56 \\
\hline 12 & 3.81 & 3.66 & 3.56 & 3.52 & 3.11 & 3.09 & 2.89 & 4.23 & 3.56 & 3.56 & 3.61 & 3.32 \\
\hline 13 & 3.61 & 3.85 & 3.45 & 3.15 & 3.25 & 3.42 & 2.98 & 3.72 & 3.67 & 3.72 & 3.91 & 3.56 \\
\hline 14 & 3.61 & 4.07 & 3.25 & 2.74 & 3.29 & 3.98 & 3.87 & 4.16 & 3.33 & 4 & 3.33 & 3.75 \\
\hline 15 & 3.81 & 3.87 & 2.82 & 2.78 & 2.69 & 3.63 & 3.66 & 3.87 & 3.33 & 3.45 & 3.72 & 3.52 \\
\hline 16 & 3.81 & 3.45 & 3.14 & 2.98 & 3.25 & 3.33 & 2.89 & 4.18 & 5.67 & 4.2 & 3.91 & 3.25 \\
\hline 17 & 3.81 & 3.52 & 3.29 & 3.18 & 3.32 & 3.36 & 2.98 & 3.96 & 4.07 & 4.03 & 3.81 & 3.32 \\
\hline 18 & 3.81 & 3.91 & 3.74 & 2.98 & 3.11 & 3.03 & 3.41 & 4.26 & 3.41 & 3.61 & 3.52 & 3.72 \\
\hline 19 & 4.12 & 3.54 & 3.42 & 2.69 & 3.72 & 3.56 & 3.66 & 3.61 & 3.38 & 3.66 & 3.18 & 3.61 \\
\hline 20 & 3.91 & 3.66 & 3.33 & 2.78 & 3.29 & 3.32 & 3.45 & 3.91 & 3.45 & 3.45 & 3.41 & 3.81 \\
\hline 21 & 3.81 & 3.56 & 3.14 & 2.69 & 3.54 & 3.98 & 4.23 & 4.07 & 3.25 & 2.89 & 3.56 & 3.45 \\
\hline 22 & 3.49 & 3.72 & 3.45 & 2.82 & 3.15 & 3.91 & 3.29 & 4.38 & 2.78 & 3.61 & 3.91 & 3.49 \\
\hline 23 & 3.36 & 3.87 & 3.42 & 2.82 & 2.89 & 3.78 & 3.45 & 3.75 & 3.72 & 3.96 & 3.81 & 3.56 \\
\hline 24 & 4 & 3.81 & 3.33 & 3.09 & 2.98 & 3.29 & 3.25 & 4.23 & 3.66 & 3.45 & 3.41 & 3.75 \\
\hline 25 & 4.12 & 3.72 & 3.36 & 3.41 & 3.03 & 2.94 & 3.41 & 4 & 3.91 & 3.45 & 3.27 & 3.45 \\
\hline 26 & 3.85 & 3.29 & 3.23 & 3.49 & 3.33 & 3.36 & 4.16 & 3.96 & 3.87 & 3.94 & 3.25 & 3.47 \\
\hline 27 & 3.91 & 3.05 & 2.89 & 3.41 & 3.09 & 3.41 & 3.75 & 3.91 & 4 & 4 & 4.07 & 3.49 \\
\hline 28 & 3.81 & 3.2 & 3.09 & 3.54 & 3.07 & 4.23 & 3.91 & 3.98 & 3.91 & 3.29 & 3.81 & 3.33 \\
\hline 29 & 3.67 & 3.56 & 2.94 & 3.36 & 3.52 & 4.38 & 3.61 & 3.85 & 4.41 & 3.83 & 3.49 & 3.52 \\
\hline 30 & 3.52 & & 2.94 & 3.23 & 3 & 3.87 & 3.52 & 4.74 & 3.45 & 3.52 & 3.56 & 3.74 \\
\hline 31 & 4 & & 3.2 & & 3.14 & & 3.52 & 4.25 & & 3.91 & & 3.66 \\
\hline
\end{tabular}


Generation of an Annual Typical Daily Wind Speed for Heights Equal and Less than 10 meters for Urban Armidale NSW, Australia

Table 9 Urban daily wind speed values obtained from Test Reference Year data for Armidale NSW, Australia at $5 \mathrm{~m}$ height in $(\mathrm{m} / \mathrm{s})$

\begin{tabular}{|c|c|c|c|c|c|c|c|c|c|c|c|c|}
\hline Day & Jan & $\mathrm{Feb}$ & Mar & Apr & May & Jun & Jul & Aug & Sep & Oct & Nov & Dec \\
\hline 1 & 3.44 & 3.87 & 3.66 & 3.07 & 3.16 & 2.82 & 3.72 & 3.47 & 3.27 & 3.72 & 3.47 & 3.27 \\
\hline 2 & 3.99 & 4.15 & 3.61 & 3.2 & 3.27 & 3.23 & 3.27 & 3.61 & 3.13 & 3.51 & 3.61 & 3.53 \\
\hline 3 & 3.44 & 3.66 & 3.61 & 2.91 & 2.99 & 2.77 & 3.2 & 4.21 & 3.91 & 3.31 & 3.72 & 3.51 \\
\hline 4 & 4.02 & 3.36 & 3.76 & 2.93 & 3.31 & 2.52 & 3.51 & 3.76 & 3.57 & 3.06 & 3.27 & 3.72 \\
\hline 5 & 3.61 & 3.1 & 3.9 & 3.47 & 2.77 & 3.13 & 3.51 & 4.02 & 3.82 & 2.97 & 3.1 & 3.99 \\
\hline 6 & 3.63 & 3.33 & 3.91 & 3.53 & 3.06 & 3.1 & 3.38 & 3.85 & 3.85 & 3.82 & 3.38 & 3.85 \\
\hline 7 & 3.99 & 3.99 & 3.51 & 3.72 & 3.16 & 2.99 & 2.91 & 4.02 & 3.51 & 3.61 & 3.38 & 3.66 \\
\hline 8 & 4.04 & 3.47 & 3.16 & 3.51 & 2.71 & 3.31 & 3.2 & 4.21 & 3.76 & 3.91 & 3.61 & 3.33 \\
\hline 9 & 4.21 & 3.76 & 3.53 & 2.97 & 2.71 & 3.57 & 3.8 & 3.76 & 3.8 & 3.8 & 3.44 & 3.23 \\
\hline 10 & 3.76 & 3.38 & 3.47 & 3.19 & 2.91 & 3.38 & 3.66 & 3.8 & 3.38 & 3.42 & 3.47 & 3.66 \\
\hline 11 & 3.9 & 3.2 & 3.51 & 2.93 & 2.97 & 3.27 & 3.66 & 3.76 & 3.7 & 3.27 & 3.38 & 3.42 \\
\hline 12 & 3.66 & 3.51 & 3.42 & 3.38 & 2.99 & 2.97 & 2.77 & 4.06 & 3.42 & 3.42 & 3.47 & 3.19 \\
\hline 13 & 3.47 & 3.7 & 3.31 & 3.03 & 3.13 & 3.29 & 2.86 & 3.57 & 3.53 & 3.57 & 3.76 & 3.42 \\
\hline 14 & 3.47 & 3.91 & 3.13 & 2.64 & 3.16 & 3.82 & 3.72 & 3.99 & 3.2 & 3.85 & 3.2 & 3.61 \\
\hline 15 & 3.66 & 3.72 & 2.71 & 2.67 & 2.58 & 3.49 & 3.51 & 3.72 & 3.2 & 3.31 & 3.57 & 3.38 \\
\hline 16 & 3.66 & 3.31 & 3.01 & 2.86 & 3.13 & 3.2 & 2.77 & 4.02 & 5.44 & 4.04 & 3.76 & 3.13 \\
\hline 17 & 3.66 & 3.38 & 3.16 & 3.06 & 3.19 & 3.23 & 2.86 & 3.8 & 3.91 & 3.87 & 3.66 & 3.19 \\
\hline 18 & 3.66 & 3.76 & 3.6 & 2.86 & 2.99 & 2.91 & 3.27 & 4.1 & 3.27 & 3.47 & 3.38 & 3.57 \\
\hline 19 & 3.96 & 3.4 & 3.29 & 2.58 & 3.57 & 3.42 & 3.51 & 3.47 & 3.25 & 3.51 & 3.06 & 3.47 \\
\hline 20 & 3.76 & 3.51 & 3.2 & 2.67 & 3.16 & 3.19 & 3.31 & 3.76 & 3.31 & 3.31 & 3.27 & 3.66 \\
\hline 21 & 3.66 & 3.42 & 3.01 & 2.58 & 3.4 & 3.82 & 4.06 & 3.91 & 3.13 & 2.77 & 3.42 & 3.31 \\
\hline 22 & 3.36 & 3.57 & 3.31 & 2.71 & 3.03 & 3.76 & 3.16 & 4.21 & 2.67 & 3.47 & 3.76 & 3.36 \\
\hline 23 & 3.23 & 3.72 & 3.29 & 2.71 & 2.77 & 3.63 & 3.31 & 3.61 & 3.57 & 3.8 & 3.66 & 3.42 \\
\hline 24 & 3.85 & 3.66 & 3.2 & 2.97 & 2.86 & 3.16 & 3.13 & 4.06 & 3.51 & 3.31 & 3.27 & 3.61 \\
\hline 25 & 3.96 & 3.57 & 3.23 & 3.27 & 2.91 & 2.82 & 3.27 & 3.85 & 3.76 & 3.31 & 3.14 & 3.31 \\
\hline 26 & 3.7 & 3.16 & 3.1 & 3.36 & 3.2 & 3.23 & 3.99 & 3.8 & 3.72 & 3.79 & 3.13 & 3.33 \\
\hline 27 & 3.76 & 2.93 & 2.77 & 3.27 & 2.97 & 3.27 & 3.61 & 3.76 & 3.85 & 3.85 & 3.91 & 3.36 \\
\hline 28 & 3.66 & 3.07 & 2.97 & 3.4 & 2.94 & 4.06 & 3.76 & 3.82 & 3.76 & 3.16 & 3.66 & 3.2 \\
\hline 29 & 3.53 & 3.42 & 2.82 & 3.23 & 3.38 & 4.21 & 3.47 & 3.7 & 4.23 & 3.68 & 3.36 & 3.38 \\
\hline 30 & 3.38 & & 2.82 & 3.1 & 2.88 & 3.72 & 3.38 & 4.55 & 3.31 & 3.38 & 3.42 & 3.6 \\
\hline 31 & 3.85 & & 3.07 & & 3.01 & & 3.38 & 4.09 & & 3.76 & & 3.51 \\
\hline
\end{tabular}



Urban Armidale NSW, Australia

Table 10 Urban daily wind speed values obtained from Test Reference Year data for Armidale NSW, Australia at $6 \mathrm{~m}$ height in $(\mathrm{m} / \mathrm{s})$

\begin{tabular}{|c|c|c|c|c|c|c|c|c|c|c|c|c|}
\hline Day & Jan & Feb & Mar & Apr & May & Jun & Jul & Aug & Sep & Oct & Nov & Dec \\
\hline 1 & 3.28 & 3.69 & 3.48 & 2.93 & 3.01 & 2.69 & 3.54 & 3.3 & 3.11 & 3.54 & 3.3 & 3.11 \\
\hline 2 & 3.8 & 3.95 & 3.43 & 3.05 & 3.11 & 3.07 & 3.11 & 3.43 & 2.98 & 3.34 & 3.43 & 3.36 \\
\hline 3 & 3.28 & 3.48 & 3.43 & 2.77 & 2.84 & 2.64 & 3.05 & 4.01 & 3.72 & 3.16 & 3.54 & 3.34 \\
\hline 4 & 3.83 & 3.2 & 3.58 & 2.79 & 3.16 & 2.4 & 3.34 & 3.58 & 3.4 & 2.91 & 3.11 & 3.54 \\
\hline 5 & 3.43 & 2.95 & 3.71 & 3.3 & 2.64 & 2.98 & 3.34 & 3.83 & 3.64 & 2.83 & 2.95 & 3.8 \\
\hline 6 & 3.46 & 3.17 & 3.72 & 3.36 & 2.91 & 2.95 & 3.22 & 3.66 & 3.66 & 3.64 & 3.22 & 3.66 \\
\hline 7 & 3.8 & 3.8 & 3.34 & 3.54 & 3.01 & 2.84 & 2.77 & 3.83 & 3.34 & 3.43 & 3.22 & 3.48 \\
\hline 8 & 3.84 & 3.3 & 3.01 & 3.34 & 2.58 & 3.16 & 3.05 & 4.01 & 3.58 & 3.72 & 3.43 & 3.17 \\
\hline 9 & 4.01 & 3.58 & 3.36 & 2.83 & 2.58 & 3.4 & 3.62 & 3.58 & 3.62 & 3.62 & 3.28 & 3.07 \\
\hline 10 & 3.58 & 3.22 & 3.3 & 3.03 & 2.77 & 3.22 & 3.48 & 3.62 & 3.22 & 3.25 & 3.3 & 3.48 \\
\hline 11 & 3.71 & 3.05 & 3.34 & 2.79 & 2.83 & 3.11 & 3.48 & 3.58 & 3.52 & 3.11 & 3.22 & 3.25 \\
\hline 12 & 3.48 & 3.34 & 3.25 & 3.22 & 2.84 & 2.83 & 2.64 & 3.87 & 3.25 & 3.25 & 3.3 & 3.03 \\
\hline 13 & 3.3 & 3.52 & 3.16 & 2.89 & 2.98 & 3.13 & 2.72 & 3.4 & 3.36 & 3.4 & 3.58 & 3.25 \\
\hline 14 & 3.3 & 3.72 & 2.98 & 2.51 & 3.01 & 3.64 & 3.54 & 3.8 & 3.05 & 3.66 & 3.05 & 3.43 \\
\hline 15 & 3.48 & 3.54 & 2.58 & 2.54 & 2.46 & 3.32 & 3.34 & 3.54 & 3.05 & 3.16 & 3.4 & 3.22 \\
\hline 16 & 3.48 & 3.16 & 2.87 & 2.72 & 2.98 & 3.05 & 2.64 & 3.83 & 5.18 & 3.84 & 3.58 & 2.98 \\
\hline 17 & 3.48 & 3.22 & 3.01 & 2.91 & 3.03 & 3.07 & 2.72 & 3.62 & 3.72 & 3.69 & 3.48 & 3.03 \\
\hline 18 & 3.48 & 3.58 & 3.43 & 2.72 & 2.84 & 2.77 & 3.11 & 3.9 & 3.11 & 3.3 & 3.22 & 3.4 \\
\hline 19 & 3.77 & 3.24 & 3.13 & 2.46 & 3.4 & 3.25 & 3.34 & 3.3 & 3.09 & 3.34 & 2.91 & 3.3 \\
\hline 20 & 3.58 & 3.34 & 3.05 & 2.54 & 3.01 & 3.03 & 3.16 & 3.58 & 3.16 & 3.16 & 3.11 & 3.48 \\
\hline 21 & 3.48 & 3.25 & 2.87 & 2.46 & 3.24 & 3.64 & 3.87 & 3.72 & 2.98 & 2.64 & 3.25 & 3.16 \\
\hline 22 & 3.2 & 3.4 & 3.16 & 2.58 & 2.89 & 3.58 & 3.01 & 4.01 & 2.54 & 3.3 & 3.58 & 3.2 \\
\hline 23 & 3.07 & 3.54 & 3.13 & 2.58 & 2.64 & 3.46 & 3.16 & 3.43 & 3.4 & 3.62 & 3.48 & 3.25 \\
\hline 24 & 3.66 & 3.48 & 3.05 & 2.83 & 2.72 & 3.01 & 2.98 & 3.87 & 3.34 & 3.16 & 3.11 & 3.43 \\
\hline 25 & 3.77 & 3.4 & 3.07 & 3.11 & 2.77 & 2.69 & 3.11 & 3.66 & 3.58 & 3.16 & 2.99 & 3.16 \\
\hline 26 & 3.52 & 3.01 & 2.95 & 3.2 & 3.05 & 3.07 & 3.8 & 3.62 & 3.54 & 3.6 & 2.98 & 3.17 \\
\hline 27 & 3.58 & 2.79 & 2.64 & 3.11 & 2.83 & 3.11 & 3.43 & 3.58 & 3.66 & 3.66 & 3.72 & 3.2 \\
\hline 28 & 3.48 & 2.93 & 2.83 & 3.24 & 2.8 & 3.87 & 3.58 & 3.64 & 3.58 & 3.01 & 3.48 & 3.05 \\
\hline 29 & 3.36 & 3.25 & 2.69 & 3.07 & 3.22 & 4.01 & 3.3 & 3.52 & 4.03 & 3.51 & 3.2 & 3.22 \\
\hline 30 & 3.22 & & 2.69 & 2.95 & 2.75 & 3.54 & 3.22 & 4.33 & 3.16 & 3.22 & 3.25 & 3.43 \\
\hline 31 & 3.66 & & 2.93 & & 2.87 & & 3.22 & 3.89 & & 3.58 & & 3.34 \\
\hline
\end{tabular}

Urban typical wind speed data is very essential for calculations concerning micro-scale wind energy generation systems and for buildings' energy calculation modelling and analysis. In this study, test reference years for mean daily wind speed for urban Armidale town NSW, Australia are generated using 20 years of the meteorologically measured data. The daily wind speed at 10 meter height for Armidale for heights [101, 9, 8, , 6, $5 \& 4]$ meter are calculated based on a numerical method utilised a previously developed meteorological test reference year annual mean daily wind speed at 10 meter height in $(\mathrm{m} / \mathrm{s})$. Those heights' TRY wind speed are presented on daily basis in a tabular form in $(\mathrm{m} / \mathrm{s})$. Results shows that urban wind speeds are relatively less than meteorological wind speeds due to the fact that there are no obstructions at meteorological weather stations.

This study provided reliable and realistic wind speed at heights range which covers the expected possible installation's heights in Armidale. It is highly recommended to base any wind energy calculations or modelling on urban wind speeds. This would reflect the real picture of urban wind potential and would save any disappointment of not reaching the expected energy generation which is usually misled by overestimation of wind speed due to the dependence on meteorological wind speed. 


\section{REFERENCES}

[1] Arens, E. A., 1981, Designing for an Acceptable Wind Speed, Transportation Engineering Journal 107, 127-141.

[2] Ansley, R. M.; W. Melbourne \& B. J. Vickery, 1977, Architectural Aerodynamics, London, applied science publishers Ltd.

[3] ASREA Handbook, 1997, Fundamentals, Chapter 15.

[4] Gandemer, 1977, Wind Environment around Buildings: Aerodynamic Concepts, Pro. Wind Effects on Buildings \& Structures, Cambridge University Press. 423-433.

[5] Givoni, Baruch, Climate Considerations in Building and Urban Design, New York, Van Nostrand Reinhold, 1998.

[6] Hunt, J. C. R., E. C. Poulton, J. C. Mumford, 1976, The Effect on Wind on People; New Criteria Based on Wind Tunnel Experiments, Building and Environment, Vol. 11, Pergamon Press, UK, pp. 15-28.

[7] Isomov, N., 1977, the Ground Level Wind Environment in Built-up Areas, Pro. Wind Effects on Buildings \& Structures, Cambridge University Press. 403-421

[8] Maklad, Y. Generation of an Annual Typical Meteorological Wind Speed for Armidale NSW, Australia. IOSR Journal of Engineering (IOSRJEN) 4(7), 2014, 9-14.

[9] J.M. Finkelstein and R.E. Schafer. Improved goodness of fit tests. Biometrika 58(3), 1971, 641-645.

[10] Tahbaz, M. The Estimation of the Wind Speed in Urban Areas. School of Architecture and Urban Planning, Shahid Beheshti University, Tehran, Iran.

[11] Penwarden, A. D. and A. F. E. Wise, Wind Environment around Buildings, London, Building Research Establishment Report, 1975. 